\title{
Effect of Nerve Growth Factor on Innervation of Perivascular Nerves in Neovasculatures of Mouse Cornea
}

\author{
Akiko Matsuyama ${ }^{a}$ Shingo Takatori, ${ }^{*, a, b}$ Yoko Sone, ${ }^{a}$ Eiko Ochi,${ }^{a}$ Mitsuhiro Goda, ${ }^{c}$ \\ Yoshito Zamami, ${ }^{a}$ Narumi Hashikawa-Hobara, ${ }^{d}$ Yoshihisa Kitamura, ${ }^{e}$ and Hiromu Kawasaki ${ }^{a, b}$ \\ ${ }^{a}$ Department of Clinical Pharmaceutical Science, Graduate School of Medicine Pharmaceutical Sciences, Okayama \\ University; 1-1-1 Tsushima-naka, Okayama 700-8530, Japan: ${ }^{b}$ Department of Clinical Pharmacy, College \\ of Pharmaceutical Sciences, Matsuyama University; 4-2 Bunkyo-cho, Matsuyama, Ehime 790-8578, Japan: \\ ${ }^{c}$ Laboratory of Biochemistry, Faculty of Pharmaceutical Sciences, Setsunan University; Hirakata, Osaka 573-0101, \\ Japan: ${ }^{d}$ Department of Life Science, Okayama University of Science; 1-1 Ridai-cho, Okayama 700-0005, Japan: and \\ ${ }^{e}$ Department of Pharmacy, Okayama University Hospital; 2-5-1 Shikata-cho, Kita-ku, Okayama 700-8558, Japan. \\ Received July 18, 2016; accepted January 11, 2017
}

Angiogenesis, which is the generation of new vascular networks from existing blood vessels, occurs under normal and pathophysiological conditions. Perivascular nerves, which innervate mature vasculatures, maintain vascular tone and regulate tissue blood flow. However, little is known whether perivascular nerves innervate newborn blood vessels. Therefore, the aim of this study was to investigate the distribution and characterization of perivascular nerves in neovasculatures, which were generated by the mouse corneal micropocket method. Under anesthesia, a pellet containing basic fibroblast growth factor (bFGF) (100 ng/pellet) was implanted into a mouse cornea in one side of the eyeball. Nerve growth factor (NGF) was locally ( 2 or $20 \mathrm{ng}$ ) applied with the pellet, or subcutaneously (40 $\mathrm{ng} / \mathrm{h}$ for $7 \mathrm{~d}$ ) administered with an osmotic mini-pump. After the implantation, vascular endothelial cells, smooth muscle cells, and perivascular nerves in the cornea were immunohistochemically studied. Neovessels generated from existing limbal vessels were observed in pellet-implanted cornea. Immunostaining of neovasculatures showed the presence of CD31-like immunoreactive (LI) endothelial cells and $\alpha$-smooth muscle actin-LI vascular smooth muscles. Perivascular nerves immunostained by protein gene product (PGP) 9.5, an axonal marker, were found in the existing limbal vessels, but they were not observed in neovasculatures. Local and subcutaneous treatment of NGF inhibits bFGF-derived angiogenesis and resulted in loop-shaped vessels that had many anastomoses, and produced innervation of PGP 9.5-LI perivascular nerves around bFGF-derived neovessels. These findings suggest that neovasculatures have no innervation of perivascular nerves, and that NGF facilitates innervations of perivascular nerves to regulate the blood flow in neovessels.

Key words nerve growth factor; perivascular nerve; vascular smooth muscle; mouse cornea

Blood vessels including arteries, arterioles, and veins, excluding capillary vessels, are innervated by perivascular nerves. It is widely accepted that perivascular nerves maintain vascular tone, and regulate organ and tissue blood flow. The sympathetic postganglionic adrenergic nerves, which mainly maintain the vascular tone, are the major efferent neural pathways to blood vessels, with few exceptions. ${ }^{1,2)}$ However, recent studies revealed that non-adrenergic non-cholinergic (NANC) perivascular nerves, including calcitonin gene-related peptide (CGRP) containing nerves (CGRPergic nerves) ${ }^{3,4)}$ and nitric oxide-containing nerves ${ }^{5,6)}$ innervate the resistance artery, such as the rat mesenteric artery, and participate in the neuronal regulation of the vascular tone.

Nerve growth factor (NGF), which promotes the innervation of central and peripheral nerves, ${ }^{7)}$ has been reported to facilitate the reinnervation of perivascular adrenergic nerves and NANC nerves in rat distal small mesenteric arteries injured by topical phenol application to the superior mesenteric artery. ${ }^{8)}$ Furthermore, our previous study demonstrated that NGF suppressed the growth of DU145 prostate carcinoma cells by increasing the number of vascular smooth muscle cells on the neovasculature in nude mice, suggesting that NGF facilitates the innervation of perivascular nerves and matures the neovasculature in tumor tissues. ${ }^{9)}$ Several reports have shown that peripheral nerves, such as the chorda tympani, lingual nerves ${ }^{10)}$ and motor neurons in hypoglossal nerves, ${ }^{11)}$ can regenerate with new processes if they are supplied with growth-promoting substrates.

The cornea of the eye has no blood vessels and maintains a degree of clearness; thus, it is suitable for observing and evaluating neovessels induced by growth factors and tumor tissues. Furthermore, it has been reported that the implantation of tumor cells into mouse and rabbit corneas results in angiogenesis. $^{12,13)}$ Since very few systematic studies have described the perivascular innervation of the microcirculation of any organ, the present study was designed to investigate whether there is perivascular innervation in neovessels derived by basic fibroblast growth factor (bFGF), and whether NGF facilitates the innervation of perivascular nerves using the mouse cornea micropocket assay.

\section{MATERIALS AND METHODS}

Animals Male BALB/c Cr Slc mice (5-7 weeks old) (purchased from Shimizu Experimental Animals, Shizuoka, Japan) were used in this study. They were housed in the Animal Research Center of Okayama University at a controlled ambient temperature of $22^{\circ} \mathrm{C}$ with $50 \pm 10 \%$ relative humidity. This study was performed in accordance with the Guidelines for Animal Experiments at the Okayama University Advanced 
Science Research Center.

Implantation of bFGF-Containing Pellets into Mouse Cornea Under sodium pentobarbital $(50 \mathrm{mg} / \mathrm{kg}$, intraperitoneally (i.p.)) anesthesia, a 1-2 mm-long incision was made in the center of the cornea in the eyeball to form a small pocket. A pellet (10- $\mu \mathrm{m}$ square) containing bFGF at a concentration of $100 \mathrm{ng} /$ pellet was then implanted into the pocket.

Pellets were formed by a gel containing Hydron (poly2-hydroxylethyl methacrylate; $12 \mathrm{mg} / \mathrm{mL}$ in $95 \%$ ethyl alcohol) (Sigma-Aldrich Japan, Tokyo, Japan), Sucralfate (sucrose octasulfate aluminum complex; $200 \mu \mathrm{g} / \mu \mathrm{L}$ in pure water) (Sigma) and bFGF $(1 \mu \mathrm{g} / \mu \mathrm{L}$ in pure water) (Sigma) medium. In the experiment for local NGF administration, pellets were formed by a gel containing both NGF at a concentration of 2 or $20 \mathrm{ng} /$ pellet and bFGF (100 ng/pellet), and implanted into the pocket made in the cornea. After the implantation, an eye-drop of $0.5 \%$ levofloxacin solution was applied to prevent infection.

NGF Administration In the experiment for local administration, NGF (TOYOBO Co., Ltd., Osaka, Japan) was applied with the implanted pellet containing bFGF and NGF ( 2 or $20 \mathrm{ng} /$ pellet). In the experiment for systemic administration, NGF was administered immediately or $3 \mathrm{~d}$ after the implantation of the bFGF-containing pellet using an osmotic mini-pump (model 1002; Alzet, Alza, Palo Alto, CA, U.S.A.) containing NGF, which had been subcutaneously implanted into the dorsal area of the mouse. NGF was continuously administered at a rate of $40 \mathrm{ng} / \mathrm{h}(48 \mu \mathrm{g} / \mathrm{kg} / \mathrm{d})$ for $7 \mathrm{~d}$.

Observation of Neovessels in the bFGF-Pellet Implanted Cornea On the 3rd, 5th, 7th, 10th, 14th, 17th, or 21st day after pellet implantation, the eyeball was exposed under ether anesthesia, and neovessels in the cornea were visually observed, and photographed with Cyber-shot 3.3 MEGA PIXELS (SONY, Tokyo, Japan). Thereafter, the length of neovessels was measured using analysis software (Hakarun), and the area of neovessels was quantitatively calculated using a formula of $\theta / 360 \times 3.1416\left[r^{2}-(r-L)^{2}\right] ; \theta$, extent angle of neovessels; $r$, radius of cornea; $L$, length of neovessels.

Immunohistochemistry Analysis The animals were anesthetized with large doses of pentobarbital-Na and sacrificed by bleeding 7, 10, 14, or $21 \mathrm{~d}$ after the implantation of the bFGF-containing pellet in an experiment to determine the time-course of angiogenesis and perivascular innervation 7 or $10 \mathrm{~d}$ after pellet implantation for the NGF treatment experiments. Thereafter, Zamboni solution (2\% paraformaldehyde and $15 \%$ picric acid in $0.15 \mathrm{~m}$ phosphate buffer) was systemically perfused via the heart, and the eyeballs were immersionfixed with Zamboni solution for $48 \mathrm{~h}$. The corneas were subsequently isolated and treated with $30 \% \mathrm{KHOH}$ solution at $60^{\circ} \mathrm{C}$ for $5 \mathrm{~min}$. After fixation, each cornea was repeatedly rinsed with phosphate buffer solution (PBS), immersed in 1\% sodium dodecyl sulfate (SDS) plus $0.5 \%$ Triton-X/PBS for $48 \mathrm{~h}$, and then incubated with PBS containing normal goat serum (GIBCOBL Co.) (1:100) for $60 \mathrm{~min}$. The corneas were incubated with the primary antibody of rabbit polyclonal anti-protein gene product (PGP) 9.5 serum $(1: 100)$ (NEO MARKERS Co.) or rat anti-CD31 serum $(1: 100)$ (SBS Co.) at $4^{\circ} \mathrm{C}$ for $72 \mathrm{~h}$. After the incubation, the corneas were washed in PBS and incubated with the secondary antibody of fluorescein-5-isothiocyanate (FITC)-labeled goat anti-rabbit immunoglobulin $\mathrm{G}$ (IgG) (diluted 1:100) (ICN Pharmaceuticals, Inc., Aurora, OH, U.S.A.) for PGP 9.5, Alexa Fluor 488-labeled goat anti-rat
$\operatorname{IgG}(1: 800)$ for CD31, or monoclonal anti- $\alpha$-smooth muscle actin ( $\alpha$-SMA) Cy3-conjugated mouse IgG (1:400) (Sigma Japan) for vascular smooth muscle at $4^{\circ} \mathrm{C}$ for $60 \mathrm{~min}$. The corneas were then thoroughly washed in PBS, mounted on slides, cover-slipped with glycerol/PBS $(2: 1 \mathrm{v} / \mathrm{v})$, and observed under a confocal laser scanning microscope (CLSM510, Carl Zeiss $\mathrm{GmbH}$, Jena, Germany). Double immunostaining of perivascular nerves and vascular smooth muscle was performed in two fluorescence views in the same microscopic field.

All data are expressed as the mean \pm standard error of the mean (S.E.M.) Statistical analyses were performed using unpaired Student's $t$-tests or an ANOVA followed by Tukey's test. $p<0.05$ was considered significant.

\section{RESULTS}

Time-Course Changes in bFGF-Induced Angiogenesis Visual observations of the eyeball showed that implantation of the pellet-containing bFGF in the cornea induced a timedependent increase in many neovessels that extended from the existing limber artery to the implanted site (Fig. 1A). As shown in Fig. 1B, the derived neovessels were constituted
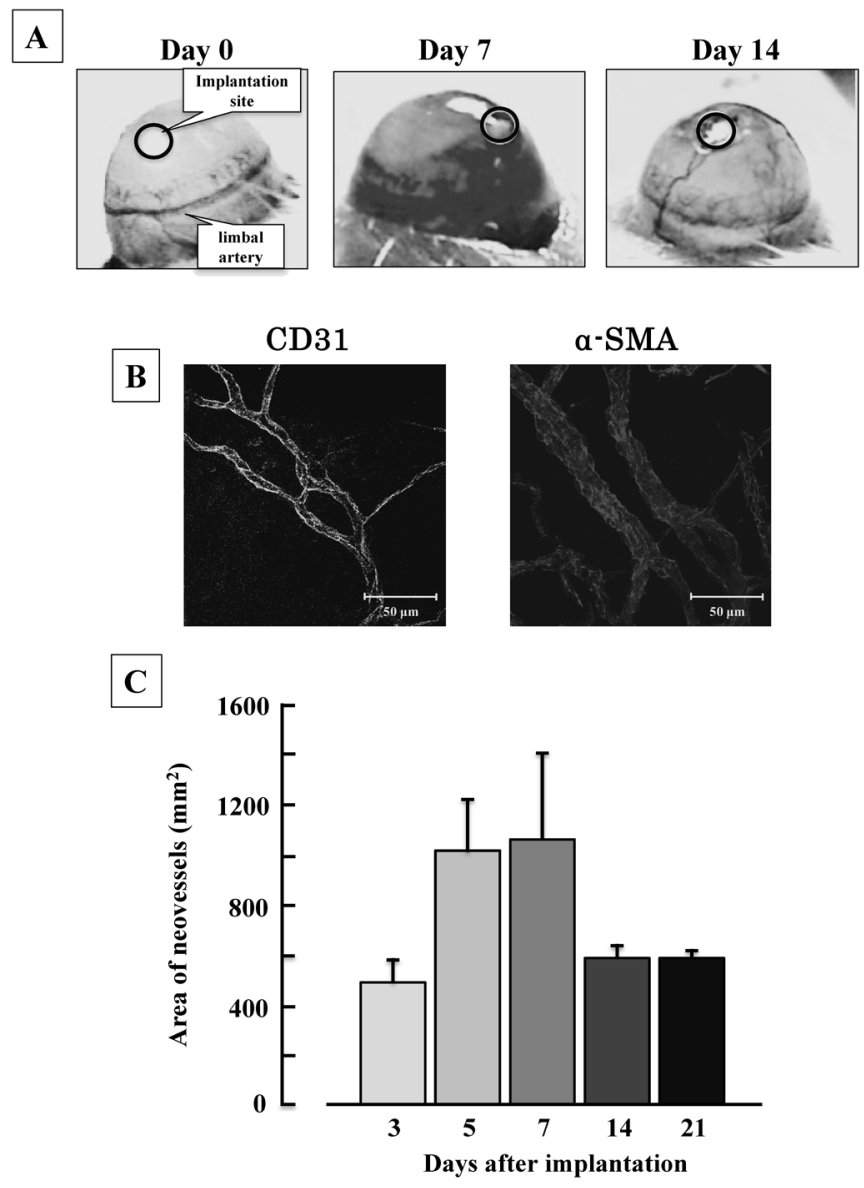

Fig. 1. Representative Images of Angiogenesis (A), Immunostained Endothelium and Vascular Smooth Muscle (B) of Neovessels, and TimeCourse Changes in Areas of Neovessels (C) in Mouse Corneas after the Implantation of Pellets Containing Basic Fibroblast Growth Factor (bFGF)

A; eyeballs before (Day 0) and after (Day 7 and 14) the pellet implantation. B; CD31-like immunoreactive (LI) endothelium and $\alpha$-smooth muscle actin (SMA)-LI cells on Day 7 after pellet implantation. C; corneal neovascular area calculated by a formula $(n=3-5)$. Each bar indicates the mean \pm S.E.M. 
with CD31-like immunoreactive (LI) endothelial cells and $\alpha$-SMA-LI cells. The area of neovessels derived by bFGF in the cornea increased time-dependently 3 to $7 \mathrm{~d}$ after pellet implantation (Fig. 1C). The increase in neovessel area reached a maximum at $7 \mathrm{~d}$ after the implantation and thereafter decreased to the levels seen at $3 \mathrm{~d}, 14$ and $21 \mathrm{~d}$ after implantation.

Distribution of PGP 9.5-Immunopositive Fibers As shown in Fig. 2, double immunostaining of $\alpha$-SMA-LI ves-
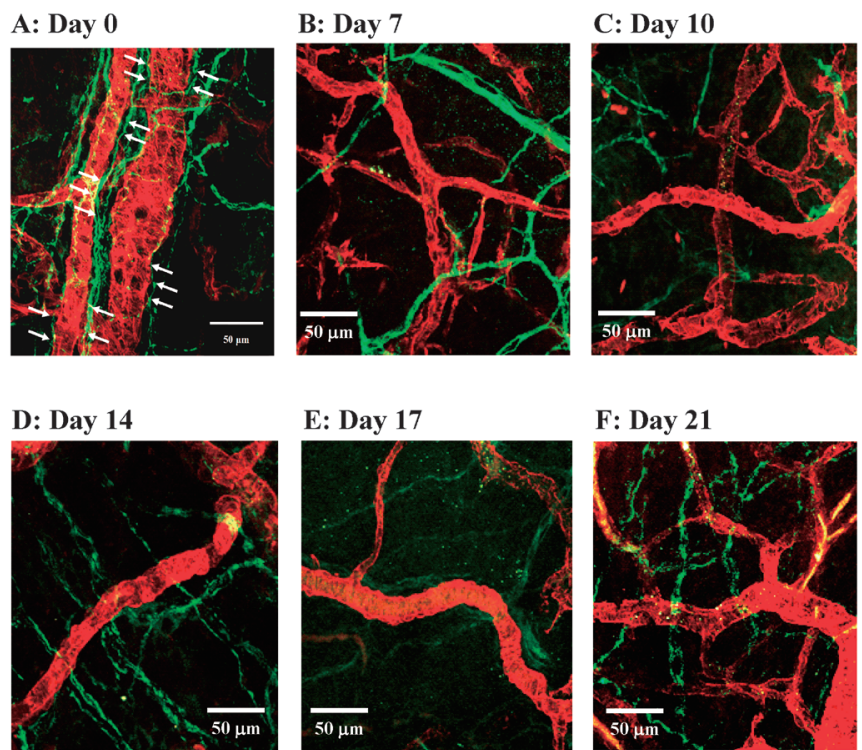

Fig. 2. Representative Double Immunostained Images Showing Protein Gene Product (PGP) 9.5-Like Immunoreactive (LI) Nerve Fibers (Green) and $\alpha$-Smooth Muscle Actin (SMA)-LI Neovessels (Red) in the Mouse Cornea 7 to $21 \mathrm{~d}$ after the Implantation of a Pellet Containing Basic Fibroblast Growth Factor (bFGF)

Arrows indicate PGP 9.5-LI fibers. sels and PGP 9.5-LI fibers of existing limbal arteries before the implantation (Fig. 2A) showed many PGP 9.5-LI fibers, most of which were in close-contact with or accompanied $\alpha$-SMA-LI vessels. A few PGP 9.5-LI fibers were observed in corneas 7 (Fig. 2B), 10 (Fig. 2C), 14 (Fig. 2D), 17 (Fig. 2E), and 21 (Fig. 2F) days after the implantation of pellets containing bFGF. However, PGP 9.5-LI fibers were distant from $\alpha$-SMA-LI neovessels and no neovessel accompanying fibers were observed (Figs. 2B-F).

Effects of NGF on the Distribution of PGP 9.5Immunopositive Fibers

Effects of Locally Administered NGF

As shown in Fig. 3A, in the corneas implanted with a pellet containing bFGF and NGF at a concentration of $20 \mathrm{ng} / \mathrm{pellet}$, many neovessels derived from the existing limber arteries (Fig. 3Da) were observed (Fig. 3Db) $7 \mathrm{~d}$ after the implantation. However, the bFGF plus NGF-induced neovessels, which did not extend and reach to the implanted site, formed a beltlike distribution around the limber arteries (Fig. 3A). Most of the neovessels formed a loop-shape (Fig. 3Dc) on the way and made several anastomoses. High concentrations of NGF (20 ng/pellet) caused angiogenesis similar to that of low concentration of NGF (2 ng/pellet). No concentration-dependent effects of NGF were observed.

In double immunostaining of $\alpha$-SMA-LI vessels (Fig. 4A) and PGP 9.5-LI fibers (Fig. 4B), a combined local application of bFGF and NGF resulted in many PGP 9.5-LI fibers (Fig. 4B), most of which accompanied $\alpha$-SMA-LI neovessels (Fig. 4C).

Effects of Systemically Administered NGF

Systemic administration of NGF for $7 \mathrm{~d}$ immediately after implantation of bFGF-containing pellet induced loop-shape neovessels, which did not reach to the implanted site (Figs. $3 \mathrm{~B}, \mathrm{Ec})$. The derived neovessels formed a belt-like distribution
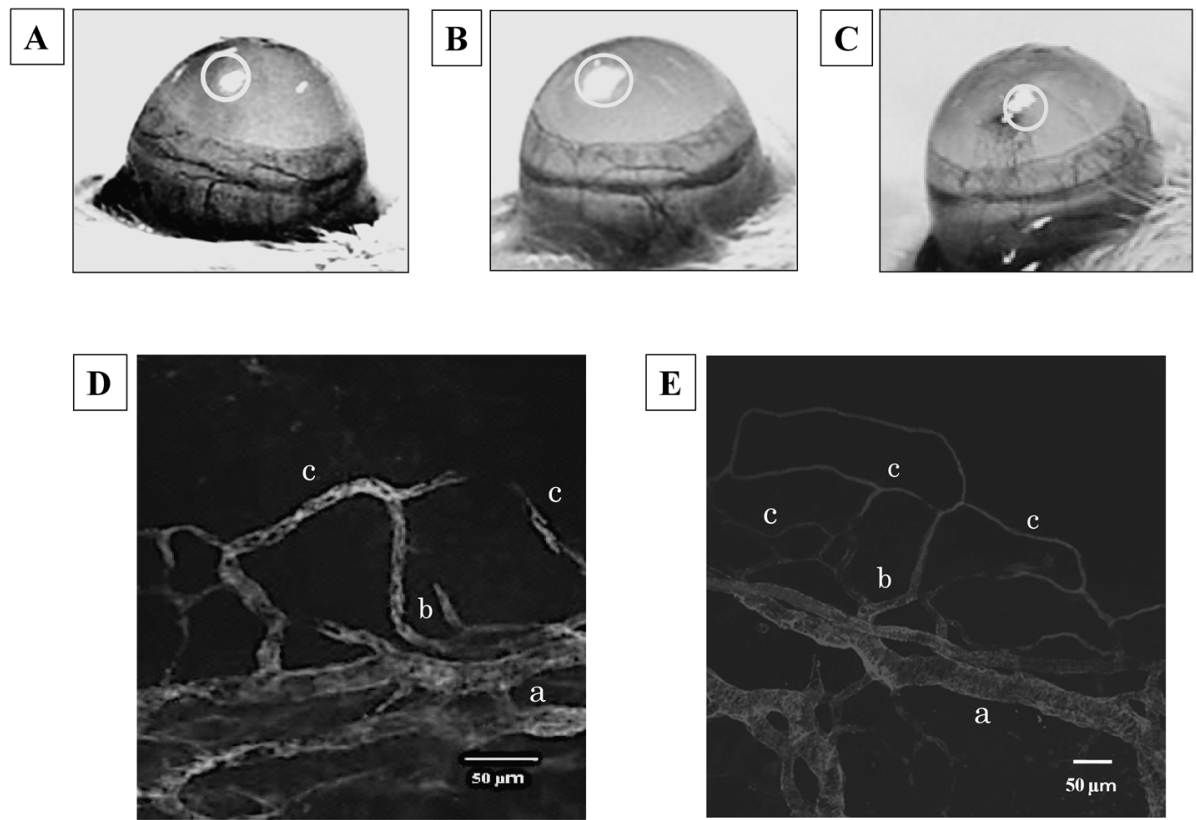

Fig. 3. Effects of Locally or Systemically Administered Nerve Growth Factor (NGF) on Basic Fibroblast Growth Factor (bFGF)-Derived Angiogenesis in the Mouse Cornea after the Implantation of a Pellet

NGF was locally (A and D) or systemically (B, C, and E) administered for $7 \mathrm{~d}$ by a pellet containing bFGF and NGF, or using an osmotic pump, respectively. A, B, and $\mathrm{C}$ show the eyeball (Day 7), immediately after and $3 \mathrm{~d}$ after the pellet implantation (Day 7) (circles), respectively. D and E show $\alpha$-smooth muscle actin (SMA)-immunopositive cells. The (a), (b), and (c) in D and E indicate the existing limber artery, derived neovessel, and loop-shaped anastomotic vessel, respectively. 


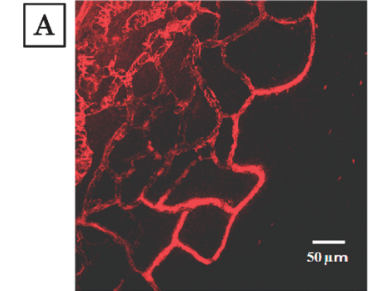

B
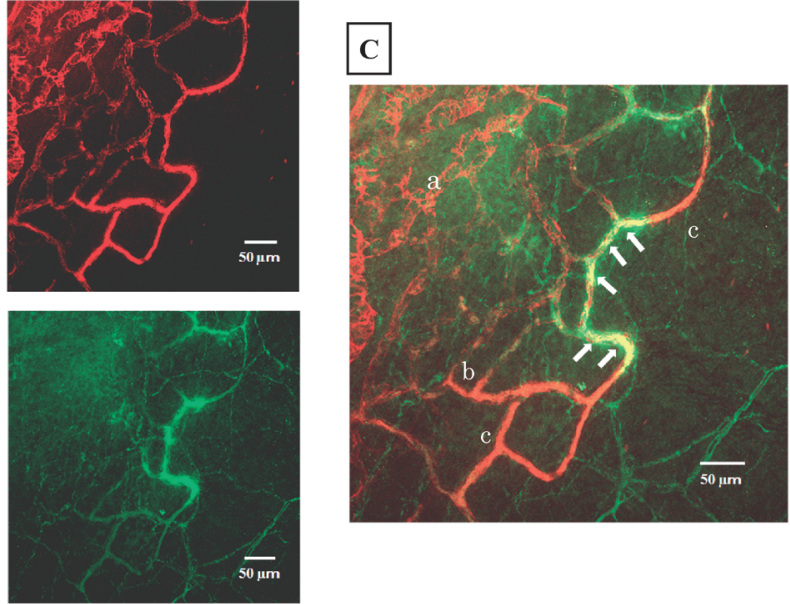

Fig. 4. Representative Images of Double Immunostained Neovessels Showing the Distribution of $\alpha$-Smooth Muscle Actin (SMA)-Like Immunoreactive (LI) Neovessels (A; Red) and Protein Gene Product (PGP) 9.5-LI Nerve Fibers (B; Green) in the Mouse Cornea Treated with Nerve Growth Factor (NGF) for $7 \mathrm{~d}$ Concomitant with Basic Fibroblast Growth Factor (bFGF)

NGF at $2 \mathrm{ng}$ /pellet was locally administered by a pellet containing bFGF and NGF. C shows a merged image of A and B. The (a), (b), and (c) in the C indicate the existing limbal artery, derived neovessel, and loop-shaped anastomotic neovessel, respectively. Arrows indicate an intense merge area.

\section{A}

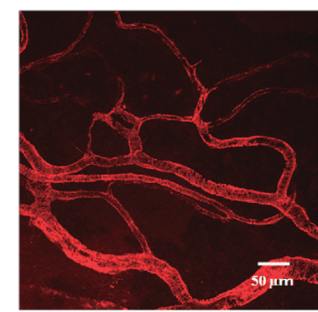

B
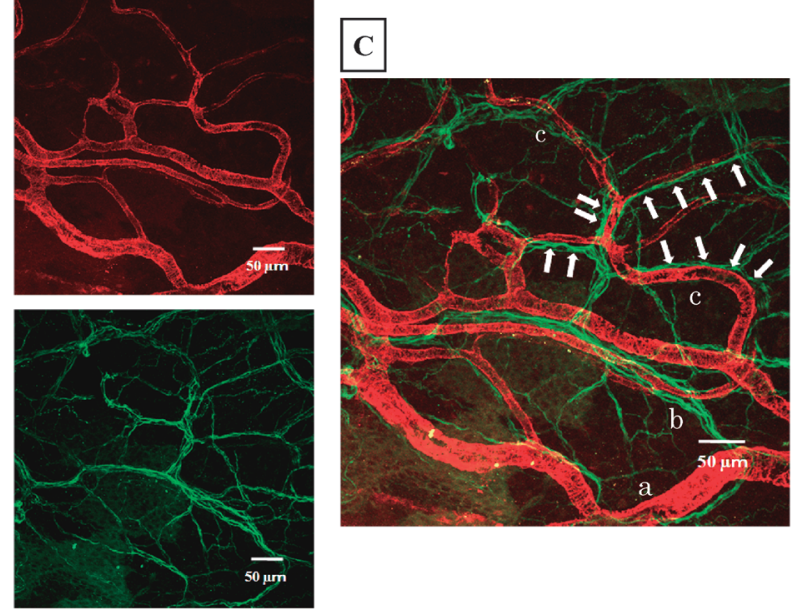

Fig. 5. Representative Images of Double Immunostained Neovessels Showing the Distribution of $\alpha$-Smooth Muscle Actin (SMA)-Like Immunoreactive (LI) Neovessels (A; Red) and Protein Gene Product (PGP) 9.5-LI Nerve Fibers (B; Green) in the Mouse Cornea Treated with Nerve Growth Factor (NGF) for $7 \mathrm{~d}$ Immediately after the Implantation of a Pellet Containing Basic Fibroblast Growth Factor (bFGF)

C shows a merged image of A and B. NGF at $40 \mathrm{ng} / \mathrm{h}$ was systemically administered by an osmotic mini-pump. The (a), (b) and (c) in C indicate the existing limber artery, derived neovessel and loop-shaped anastomotic neovessel, respectively. Arrows indicate PGP 9.5-LI fibers.

and stayed around the existing limber arteries, as observed in the corneas with locally applied NGF. Most of the neovessels derived by bFGF were anastomotic vessels with systemically NGF treatment (Figs. 3B, 5A). In double immunostaining of $\alpha$-SMA-LI neovessels and PGP 9.5-LI fibers, a 7-d treatment with NGF immediately after the implantation of bFGF resulted in many PGP 9.5-LI fibers (Fig. 5B), most of which accompanied $\alpha$-SMA-LI neovessels (Fig. 5C).

Small numbers of neovessels extending to the implanted
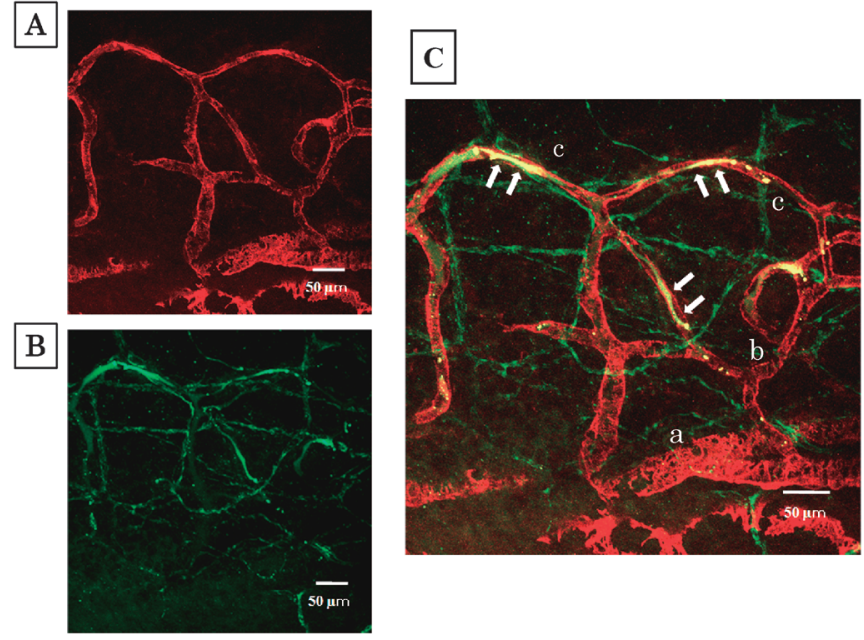

Fig. 6. Representative Images of Double Immunostained Neovessels Showing the Distribution of $\alpha$-Smooth Muscle Actin (SMA)-Like Immunoreactive (LI) Neovessels (A; Red) and Protein Gene Product (PGP) 9.5-LI Nerve Fibers (B; Green) in the Mouse Cornea Treated with Nerve Growth Factor (NGF) for $7 \mathrm{~d}$ from $3 \mathrm{~d}$ after the Implantation of a Pellet Containing Basic Fibroblast Growth Factor (bFGF)

NGF at $40 \mathrm{ng} / \mathrm{h}$ was systemically administered by an osmotic mini-pump. $\mathrm{C}$ shows a merged image of $\mathrm{A}$ and $\mathrm{B}$. The (a), (b) and (c) in $\mathrm{C}$ indicate the existing limber artery, derived neovessel and loop-shaped anastomotic neovessel, respectively. Arrows indicate an intense merge area.

site of the bFGF-containing pellet were observed in the corneas that were systemically treated with NGF for $7 \mathrm{~d}$ from $3 \mathrm{~d}$ after the implantation of the pellet (Fig. 3C). However, most of the neovessels derived by bFGF in the systemically administered NGF were loop-shaped and anastomotic vessels (Fig. 6A).

A large number of PGP 9.5-LI fibers were observed in corneas treated with NGF for $7 \mathrm{~d}$ from $3 \mathrm{~d}$ after the implantation of the bFGF-containing pellet (Fig. 6B). PGP 9.5-LI fibers were in close contact with $\alpha$-SMA-LI neovessel (Fig. 6C).

\section{DISCUSSION}

The present study demonstrated that when bFGF-containing pellets were implanted in mouse corneas, neovessels were generated from the existing limbal arteries to the implanted site. Additionally, the immunohistochemistry analysis confirmed that neovessels generated by the cornea micropocket method were constituted with endothelium cells and vascular smooth muscle cells, indicating that neovessels derived by bFGF are as functionally-efficient as a blood vessel. The neovessels induced after the implantation of a bFGF-containing pellet increased in a time-dependent manner, and reached a maximum, and thereafter decreased to the initial levels. This may be due to a decreased concentration of bFGF in the pellet and/or an apoptosis phenomenon.

The immunohistochemical studies using the neuroaxonal marker, PGP 9.5 revealed many PGP 9.5-LI fibers, which accompanied $\alpha$-SMA-LI vessels in the existing limbal arteries in mouse corneas. Although PGP 9.5-LI fibers exists around neovessels in the cornea, they were sparse and distant from $\alpha$-SMA-LI vessels and were not in contact close with neovessels even $21 \mathrm{~d}$ after the implantation of a bFGF-containing pellet. These findings suggest that these neovessels had little, if any, perivascular innervation. The main reason for the lack 
of a nervous system in neovessels may be that the cornea contains inhibitory substances, such as semaphorin $3 \mathrm{~A}$ and proteoglycan chondroitin sulfate, ${ }^{14)}$ which prevent the distribution of perivascular nerves. ${ }^{15-17)}$ It is suggested that bFGF increases semaphorin $3 \mathrm{~A}$ expression to inhibit perivascular innervation, as reported by a study that expression of semaphorin $3 \mathrm{~A}$ in corneal fibroblasts is regulated by epidermal growth factor released from corneal epithelial cells. ${ }^{18)}$

The present study demonstrated that the NGF treatment immediately after the implantation of pellets, regardless of local or systemic administration, inhibited the extension of neovessels reaching the bFGF-implanted site and stayed neovessels around the existing limber arteries. In immunohistochemistry studies, the morphological characteristics of derived neovessels, anastomosis and a loop-shape vessel formation was found in the NGF-treated cornea. These findings strongly suggest that NGF inhibits bFGF-induced angiogenesis via morphological changes of neovessels. It is highly possible that NGF may inhibit growth or migration of endothelial cells derived by bFGF in the cornea. This hypothesis is also supported by the present findings, that bFGF could not induce further angiogenesis after NGF administration, though bFGF derived neovessels before NGF treatment. NGF treatments have been reported to enhance the migration of human lung fibroblasts as well as human vascular smooth muscle cells. ${ }^{19-21)}$ Thus, NGF may promote the migration of vascular smooth muscle cells to tumor neovessels and mature vessels. Human and rat vascular smooth muscle cells express the NGF receptor, trk A. ${ }^{21)}$ In addition, NGF-induced smooth muscle cell migration is similar to the migratory effects obtained in vitro with platelet-derived growth factor-BB. ${ }^{22)}$ Therefore, NGF appears to be a potent chemoattractant for vascular smooth muscle cells. Furthermore, in the present study, perivascular nerves, which were in close contact with and accompanied neovessels derived by bFGF, were clearly observed after treatment with NGF. Although the present study did not investigate the localization of CD31-LI endothelial cells and PGP 9.5-LI fibers, PGP 9.5-LI fibers have been reported to distribute mainly to $\alpha$-SMA-LI vascular cells, but not CD31-LI endothelial cells. ${ }^{23,24)}$ This is also supported by previous reports that neovasculatures derived tumor are consisted of endothelium-like and immature vessels without a coating of smooth muscle cells, and they are not innervated or exhibit the progressive loss of perivascular innervation. ${ }^{25-27)} \mathrm{A}$ previous study reported that peripheral nerves provide a template that determines the organotypical pattern of blood vessel branching and arterial differentiation in the skin. ${ }^{28)}$ We previously reported that NGF suppressed the tumor growth of human prostate cancer cells (DU145) in the nude mouse. ${ }^{9)}$ Therefore, NGF facilitates the maturation of tumor neovessels by migrating smooth muscle cells via innervation of perivascular nerves, which may regulate blood flow to tumor tissues and results in the suppression of tumor growth. It seems likely that inhibition of semaphorin 3A may be the possible mechanism of NGF-induced facilitatory effect on perivascular innervation of neovessels, as reported by Kaselis et al. that the increased NGF concentrations inhibited semaphorin 3A-induced inhibitory effect on DRG axon outgrowth. ${ }^{29)}$ However, a further study is needed to clarify this mechanism.

In conclusion, the present results suggest that NGF facilitates the innervation of perivascular nerves in order to mature neovessels and regulate blood flow. It is hypothesized that NGF may have anti-tumor activity by accelerating the maturation of the neovasculature in tumor tissues, which are frangible and unmature, thereby inhibiting tumor growth.

Acknowledgments We appreciate the kind support from Dr. Sadaaki Maeda and Dr. Jyunji Kasai, Department of Pharmacotherapeutics, Faculty of Pharmaceutical Sciences, Setsunan University, to give us the experimental skills for the mouse corneal micropocket method.

Conflict of Interest The authors declare no conflict of interest.

\section{REFERENCES}

1) Montastruc JL, Rascol O, Senard JM. The discovery of vasomotor nerves. Clin. Auton. Res., 6, 183-187 (1996).

2) Coote JH. Landmarks in understanding the central nervous control of the cardiovascular system. Exp. Physiol., 92, 3-18 (2007).

3) Takatori S, Hirai K, Ozaki S, Tangsucharit P, Fukushima-Miyashita S, Goda M, Hashikawa-Hobara N, Ono N, Kawasaki H. Protons modulate perivascular axo-axonal neurotransmission in the rat mesenteric artery. Br. J. Pharmacol., 171, 5743-5756 (2014).

4) Takatori S, Fujiwara H, Zamami Y, Hashikawa-Hobara N, Kawasaki H. Decreased perivascular CGRP-containing nerves in Otsuka Long-Evans Tokushima Fatty rats with insulin resistance and hypertension. Hypertens. Res., 37, 398-404 (2014).

5) Hatanaka Y, Hobara N, Honghua J, Akiyama S, Nawa H, Kobayashi Y, Takayama F, Gomita Y, Kawasaki H. Neuronal nitric-oxide synthase inhibition facilitates adrenergic neurotransmission in rat mesenteric resistance arteries. J. Pharmacol. Exp. Ther., 316, 490-497 (2006).

6) Yokomizo A, Takatori S, Hashikawa-Hobara N, Goda M, Kawasaki $\mathrm{H}$. Characterization of perivascular nerve distribution in rat mesenteric small arteries. Biol. Pharm. Bull., 38, 1757-1764 (2015).

7) Korsching S, Thoenen H. Nerve growth factor in sympathetic ganglia and corresponding target organs of the rat: correlation with density of sympathetic innervation. Proc. Natl. Acad. Sci. U.S.A., 80, 3513-3516 (1983).

8) Yokomizo A, Takatori S, Hashikawa-Hobara N, Goda M, Kawasaki $\mathrm{H}$. Nerve growth factor facilitates redistribution of adrenergic and non-adrenergic non-cholinergic perivascular nerves injured by phenol in rat mesenteric resistance arteries. Eur. J. Pharmacol., 770, 110-116 (2016).

9) Goda M, Atagi S, Amitani K, Hobara N, Kitamura Y, Kawasaki H. Nerve growth factor suppresses prostate tumor growth. J. Pharmacol. Sci., 112, 463-466 (2010).

10) Smith KG, Yates JM, Robinson PP. The effect of nerve growth factor on functional recovery after injury to the chorda tympani and lingual nerves. Brain Res., 1020, 62-72 (2004).

11) Fan LY, Wang ZC, Wang P, Lan YY, Tu L. Exogenous nerve growth factor protects the hypoglossal nerve against crush injury. Neural Regen. Res., 10, 1982-1988 (2015).

12) Gimbrone MA Jr, Cotran RS, Leapman SB, Folkman J. Tumor growth and neovascularization: an experimental model using the rabbit cornea. J. Natl. Cancer Inst., 52, 413-427 (1974).

13) Muthukkaruppan VR, Kubai L, Auerbach R. Tumor-induced neovascularization in the mouse eye. J. Natl. Cancer Inst., 69, 699-708 (1982).

14) Sango K, Oohira A, Ajiki K, Tokashiki A, Horie M, Kawano H. Phosphacan and neurocan are repulsive substrata for adhesion and neurite extension of adult rat dorsal root ganglion neurons in vitro. Exp. Neurol., 182, 1-11 (2003).

15) Herman JG, Meadows GG. Increased class 3 semaphorin expression 
modulates the invasive and adhesive properties of prostate cancer cells. Int. J. Oncol., 30, 1231-1238 (2007).

16) Vachkov IH, Huang X, Yamada $Y$, Tonchev AB, Yamashima $T$, Kato $\mathrm{S}$, Takakura N. Inhibition of axonal outgrowth in the tumor environment: involvement of class 3 semaphorins. Cancer Sci., 98, 1192-1197 (2007).

17) Capparuccia L, Tamagnone L. Semaphorin signaling in cancer cells and in cells of the tumor microenvironment - two sides of a coin. $J$. Cell Sci., 122, 1723-1736 (2009).

18) Ko JA, Morishige N, Yanai R, Nishida T. Up-regulation of semaphorin $3 \mathrm{~A}$ in human corneal fibroblasts by epidermal growth factor released from cocultured human corneal epithelial cells. Biochem. Biophys. Res. Commun., 377, 104-108 (2008).

19) Kohyama T, Liu X, Wen FQ, Kobayashi T, A be S, Ertl R, Rennard SI. Nerve growth factor stimulates fibronectin-induced fibroblast migration. J. Lab. Clin. Med., 140, 329-335 (2002).

20) Micera A, Vigneti E, Pickholtz D, Reich R, Pappo O, Bonini S, Maquart FX, Aloe L, Levi-Schaffer F. Nerve growth factor displays stimulatory effects on human skin and lung fibroblasts, demonstrating a direct role for this factor in tissue repair. Proc. Natl. Acad. Sci. U.S.A., 98, 6162-6167 (2001).

21) Donovan MJ, Miranda RC, Kraemer R, McCaffrey TA, Tessarollo L, Mahadeo D, Sharif S, Kaplan DR, Tsoulfas P, Parada L, Toran-Allerand CD, Hajjar DP, Hempstead BL. Neurotrophin and neurotrophin receptors in vascular smooth muscle cells. Regulation of expression in response to injury. Am. J. Pathol., 147, 309-324 (1995).

22) Kraemer R, Nguyen H, March KL, Hempstead B. NGF activates similar intracellular signaling pathways in vascular smooth muscle cells as PDGF-BB but elicits different biological responses. Arterioscler. Thromb. Vasc. Biol., 19, 1041-1050 (1999).

23) Goda M, Takatori S, Atagi S, Hashikawa-Hobara N, Kawasaki H. Nerve growth factor facilitates perivascular innervation in neovasculatures of mice. J. Pharmacol. Sci., 131, 251-258 (2016).

24) Sone Y, Takatori S, Ochi E, Zamami Y, Matsuyama A, Fukuhara S, Goda M, Kitamura Y, Kawasaki H. Nerve growth factor facilitates the innervation of perivascular nerves in tumor-derived neovasculature in the mouse cornea. Pharmacology, 99, 57-66 (2017).

25) Chamary VL, Robson T, Loizidou M, Boulos PB, Burnstock G. Progressive loss of perivascular nerves adjacent to colorectal cancer. Eur. J. Surg. Oncol., 26, 588-593 (2000).

26) Morikawa S, Baluk P, Kaidoh T, Haskell A, Jain RK, McDonald DM. Abnormalities in pericytes on blood vessels and endothelial sprouts in tumors. Am. J. Pathol., 160, 985-1000 (2002).

27) Abramsson A, Lindblom P, Betsholtz C. Endothelial and nonendothelial sources of PDGF-B regulate pericyte recruitment and influence vascular pattern formation in tumors. J. Clin. Invest., 112, $1142-1151$ (2003).

28) Mukouyama YS, Shin D, Britsch S, Taniguchi M, Anderson DJ. Sensory nerves determine the pattern of arterial differentiation and blood vessel branching in the skin. Cell, 109, 693-705 (2002).

29) Kaselis A, Treinys R, Vosyliūtė R, Satkauskas S. DRG axon elongation and growth cone collapse rate induced by Sema3A are differently dependent on NGF concentration. Cell. Mol. Neurobiol., 34, 289-296 (2014). 